\title{
Progrès et menaces
}

Gérard Friedlander, François A. Auger

> Comme chaque année, la trêve des Fêtes de fin d'année est aussi l'occasion de faire le point. L'année qui vient de s'achever a été pour notre revue une année d'évolution, marquée par d'importants changements. Le contenu tout d'abord : malgré une diminution du nombre de pages de 128 à 112 à partir d'avril, le nombre d'articles de synthèse a encore augmenté par rapport à 2002 et 2003, pour s'approcher de la centaine. L'objectif était simple : accroître la diversité des sujets traités et présenter ceux-ci de manière plus concise. Dans le même temps, un travail de fond sur les illustrations (figures et tableaux, encadrés) a été mené afin de rendre celles-ci plus homogènes, plus faciles à utiliser, attirantes, voire même plus éloquentes.

Comment ces inflexions ont-elles été perçues? Les indicateurs de satisfaction ou d'attractivité sont difficiles à définir et leur maniement ne peut être que prudent. Deux éléments positifs sont néanmoins à relever : d'abord le nombre d'abonnés, qui approche de cinq mille; leur typologie évolue car les étudiants, toujours plus nombreux, en représentent maintenant plus de la moitié. Ensuite les auteurs: formidablement réactifs lorsqu'ils sont sollicités, ils soumettent de plus en plus souvent, spontanément, des articles de synthèse.

À en croire les statistiques de fréquentation, le site de médecine/sciences est devenu un outil au quotidien pour nombre de chercheurs avec une moyenne journalière de près de 2000 requêtes de pages.

Au plan scientifique et médical, 2004 a apporté son lot d'émotions, d'innovations et de drames. La petite dame de Flores, hominidé distinct d'Homo sapiens, aurait vécu jusqu'à il y a 18000 ans; un embryon humain a été obtenu par transfert nucléaire; I'ADN non codant, qui représente plus de $90 \%$ du génome, commence à livrer ses secrets, et son rôle dans l'évolution semble fondamental; après l'apoptose, l'autophagie suscite de plus en plus de travaux et la lutte pharmacologique contre l'obésité s'intensifie.

Le front des maladies transmissibles, fléaux d'hier et d'aujourd'hui, est loin d'être serein. Le SRAS à peine calmé, la grippe aviaire menace, tandis que le sida, loin d'être écrit au passé, connaît un regain d'activité sur tous les continents, dans un contexte d'enjeux socio-économiques surtout en ce qui a trait au continent africain.

Deux mille cinq sera l'année du vingtième anniversaire de notre revue. Les rédacteurs en chef qui ont animé médecine/sciences depuis sa création sauront évoquer les moments exaltants de ces deux décennies. Suivant les suggestions de nos lecteurs, nous proposerons au cours de l'année une alternance de numéros «classiques» et de numéros à thème, abordant l'épigénétique, le vieillissement, la biologie de la peau ou le repliement des protéines. Nous espérons que ces numéros sauront vous plaire et constituer des documents de référence, tâche toujours plus ardue au fur et à mesure que l'accumulation des connaissances s'accélère.

Notre plaisir à remercier auteurs, lecteurs, évaluateurs, ainsi que les équipes de rédaction et d'édition, est chaque année aussi grand.

Que l'année qui débute apporte à chacun de vous ce qu'il désire et au monde un peu de paix et de tolérance. $\diamond$

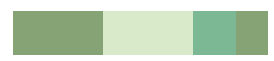

Gérard Friedlander

Rédacteur en chef France
François A. Auger Rédacteur en chef Québec 